JOURNAL OF

FUNCTION SPACES AND APPLICATIONS

Volume 8, Number 2 (2010), 201-213 (c) 2010, Scientific Horizon

http://www.jfsa.net

\title{
On Hilbert extensions of Weierstrass' theorem with weights
}

\author{
Yamilet Quintana ${ }^{1}$ \\ (To Maíta, in memoriam) \\ (Communicated by Wilfredo Urbina)
}

2000 Mathematics Subject Classification. Primary 41, 41A10; Secondary 43A32, $47 \mathrm{~A} 56$.

Keywords and phrases. Weierstrass' theorem, $\mathcal{G}$-valued weights, $\mathcal{G}$-valued polynomials, $\mathcal{G}$-valued continuous functions.

\footnotetext{
Abstract. In this paper we study the set of $\mathcal{G}$-valued functions which can be approximated by $\mathcal{G}$-valued continuous functions in the norm $L_{\mathcal{G}}^{\infty}(I, w)$, where $I \subset \mathbb{R}$ is a compact interval, $\mathcal{G}$ is a separable real Hilbert space and $w$ is a certain $\mathcal{G}$-valued weakly measurable weight. Thus, we obtain a new extension of the celebrated Weierstrass approximation theorem.
}

\section{Introduction}

If $I \subset \mathbb{R}$ is any compact interval, Weierstrass' approximation theorem says that $C(I)$ is the largest set of functions which can be approximated by polynomials in the norm $L^{\infty}(I)$, if we identify, as usual, functions which are equal almost everywhere. Weierstrass proved this theorem in 1885 . He also proved the density of trigonometric polynomials in the class of $2 \pi$ periodic continuous real-valued functions. These results were, in a sense, a counterbalance to Weierstrass' famous example given in 1861 about the existence of a continuous nowhere differentiable function (see [1]).

\footnotetext{
${ }^{1}$ Research partially supported by DID-USB under Grant DI-CB-015-04
} 
The result obtained in his paper in 1885 titled On the possibility of giving an analytic representation to an arbitrary function of real variable [15], shows that he suspected that any analytic functions could be represented by power series. Weierstrass' approximation theorem can be stated as follows.

Theorem 1.1. (K. Weierstrass) Given $f:[a, b] \rightarrow \mathbb{R}$ continuous and an arbitrary $\epsilon>0$, there exists an algebraic polynomial $p$ such that

$$
|f(x)-p(x)| \leq \epsilon, x \in[a, b] .
$$

There have been many improvements, generalizations and extensions of this theorem; such results may be found in [29], [3], [6], [11] and [12]. Further, we should recall the Bernstein's problem on approximation by polynomials on the whole real line (see [15], [16] and [17]), and the approximation problem for unbounded functions in $I$ (see for example, [10]).

Some recent generalizations of Weierstrass' approximation theorem use weighted approximation. More precisely, if $I \subset \mathbb{R}$ is a compact interval, the approximation problem is studied with the norm $L^{\infty}(I, w)$ defined by

$$
\|f\|_{L^{\infty}(I, w)}:=\operatorname{ess}_{\sup } \operatorname{su}_{x \in I}|f(x)| w(x),
$$

where $w$ is a weight, i.e., a non-negative measurable function. The convention $0 \cdot \infty=0$ is used as well. Observe that (1.2) is not the usual definition of the $L^{\infty}$ norm in the context of measure theory, although it is the correct definition when we work with weights (see e.g. [4] and [7]). The reader may find in [23], [25], [26] and [27] recent and detailed results on this subject.

Other kinds of approximation problems can arise when we consider simultaneous approximation including derivatives of certain functions; this is the case for Weierstrass' theorem in the context of weighted Sobolev spaces. About this subject we refer to [25] and [26].

In this paper we give a new result on Weierstrass' approximation theorem with weights in Hilbert spaces. The paper is organized as follows. In Section 2 we provide some notation, necessary preliminaries and auxiliary results which will be often used throughout the text. Usually we shall use standard notation, and it will be properly introduced whenever needed. In Section 3 we present the main result about approximation in $L_{\mathcal{G}}^{\infty}(I, w)$.

\section{Preliminaries}

In what follows, $I$ stands for any compact interval in $\mathbb{R}$. By $l^{2}(\mathbb{R})$ we denote the real linear space of all sequences $\left\{x_{n}\right\}_{n \in \mathbb{Z}_{+}}$with 
$\sum_{n=0}^{\infty}\left|x_{n}\right|^{2}<\infty$, and $\left(\mathcal{G},\langle\cdot, \cdot\rangle_{\mathcal{G}}\right)$ stands for a separable real Hilbert space with associated norm denoted by $\|\cdot\|_{\mathcal{G}}$.

It is well-known that every real and separable Hilbert space $\mathcal{G}$ is isomorphic either to $\mathbb{R}^{n}$ for some $n \in \mathbb{N}$ or to $l^{2}(\mathbb{R})$. In each case, $\mathcal{G}$ has the structure of a commutative Banach algebra with the coordinatewise operations. In the first case, we have a commutative Banach algebra with identity and the second case, a commutative Banach algebra without identity. The reader is referred to [8], [13] or [32] for more details about these statements.

However, in practice the representation which we obtain by means of this isomorphism is not always interesting, because the properties of the individual elements of $\mathcal{G}$ can be in many cases more fruitful. This happens when $\mathcal{G}$ is a Hilbert space of analytic functions or differentiable functions. Despite this, it is very valuable to know the representation given by this isomorphism because it allows us to determine how useful the properties of the Hilbert space by itself can be.

2.1. On weighted spaces. A detailed discussion about properties of weighted spaces may be found in [5], [9], [14], [18] or [22]. We recall here some important tools and definitions which will be used throughout this paper.

Definition 2.1. A scalar weight $w$ is a measurable function $w: \mathbb{R} \rightarrow$ $[0, \infty]$. If $w$ is only defined in $A \subset \mathbb{R}$, we set $w:=0$ in $\mathbb{R} \backslash A$.

Definition 2.2. Given a measurable set $A \subset \mathbb{R}$ and a scalar weight $w$, we define the space $L^{\infty}(A, w)$ as the space of equivalence classes of measurable functions $f: A \longrightarrow \mathbb{R}$ with respect to the norm

$$
\|f\|_{L^{\infty}(A, w)}:=\operatorname{ess} \sup _{x \in A}|f(x)| w(x) .
$$

This space inherits some properties from the classical Lebesgue space $L^{\infty}(A)$ and it allows us to study new functions, which do not fit in the classical $L^{\infty}(A)$ (see, for example [5], [28]). Other properties of $L^{\infty}(A, w)$ have a strong relation with the nature of the weight $w$ : in fact, if $A=I$ and $w$ has a multiplicative inverse, (i.e. there exists a weight $w^{-1}: I \longrightarrow \mathbb{R}$, such that $\left.w(t) w^{-1}(t)=1, \quad t \in I\right)$ then, it is easy to see that $L^{\infty}(I, w)$ and $L^{\infty}(I)$ are isomorphic, since the map $\Psi_{w}: L^{\infty}(I, w) \rightarrow L^{\infty}(I)$ given by $\Psi_{w}(f)=f w$ is a linear and bijective isometry, and therefore, $\Psi_{w}$ is also homeomorphism, or equivalently, for all $Y \subseteq L^{\infty}(I, w)$, we have $\Psi_{w}(\bar{Y})=\overline{\Psi_{w}(Y)}$, where we take each closure with respect to the norms $L^{\infty}(I, w)$ and $L^{\infty}(I)$, respectively. Also, for all $A \subseteq L^{\infty}(I)$, $\Psi_{w}^{-1}(\bar{A})=\overline{\Psi_{w}^{-1}(A)}$ and $\Psi_{w}^{-1}=\Psi_{w^{-1}}$. Then using Weierstrass' theorem 
we have,

$$
\Psi_{w}^{-1}(\overline{\mathbb{P}})=\overline{\Psi_{w}^{-1}(\mathbb{P})}=\left\{f \in L^{\infty}(I, w): f w \in C(I)\right\} .
$$

Unfortunately, the last equality in (2.3) does not allow us to obtain information on local behavior of the functions $f \in L^{\infty}(I, w)$ which can be approximated. Furthermore, if $f \in L^{\infty}(I, w)$, then in general $f w$ is not a continuous function, since its continuity also depends of the singularities of weight $w$ (see [5], [15], [16], [27], [23], [26]).

The next definition presents the classification of the singularities of a scalar weight $w$ done in [26] to show the results about density of continuous functions in the space $L^{\infty}(\operatorname{supp}(w), w)$.

Definition 2.3. Given a scalar weight $w$ we say that $a \in \operatorname{supp}(w)$ is a singularity of $w$ (or singular for $w$ ) if

$$
\operatorname{ess} \lim \inf _{x \in \operatorname{supp}(w), x \rightarrow a} w(x)=0 .
$$

We say that a singularity $a$ of $w$ is of type 1 , type 2 or type 3 if, respectively, ess $\lim _{x \rightarrow a} w(x)=0,0<\operatorname{ess} \lim \sup _{x \rightarrow a} w(x)<\infty$ or ess lim $\sup _{x \rightarrow a} w(x)=\infty$.

We denote by $S$ and $S_{i}(i=1,2,3)$ respectively, the set of singularities of $w$ and the set of singularities of $w$ of type $i$.

We say that $a \in S_{i}^{+}$(respectively $a \in S_{i}^{-}$) if $a$ verifies the property in the definition of $S_{i}$ when we take the limit as $x \rightarrow a^{+}$(respectively $x \rightarrow a^{-}$). We define $S^{+}:=S_{1}^{+} \cup S_{2}^{+} \cup S_{3}^{+}$and $S^{-}:=S_{1}^{-} \cup S_{2}^{-} \cup S_{3}^{-}$.

Definition 2.4. Given a scalar weight $w$, we define the right regular and left regular points of $w$, respectively, as

$$
\begin{aligned}
& R^{+}:=\left\{a \in \operatorname{supp}(w): \operatorname{ess} \lim \inf _{x \in \operatorname{supp}(w), x \rightarrow a^{+}} w(x)>0\right\} \\
& R^{-}:=\left\{a \in \operatorname{supp}(w): \operatorname{ess} \lim \inf _{x \in \operatorname{supp}(w), x \rightarrow a^{-}} w(x)>0\right\} .
\end{aligned}
$$

The following result was proved in [26] and it states a characterization for the functions in $L^{\infty}(\operatorname{supp}(w), w)$ which can be approximated by continuous functions in norm $L^{\infty}(\operatorname{supp}(w), w)$ for every $w$.

Theorem 2.1. (Portilla et al. [[26], Theorem 1.2]). Let $w$ be any scalar weight and

$$
H_{0}:=\left\{\begin{array}{c}
f \in L^{\infty}(\operatorname{supp}(w), w): \\
f \text { is continuous to the right at every point of } R^{+}, \\
f \text { is continuous to the left at every point of } R^{-}, \\
\text {for each } a \in S^{+}, \text {ess } \lim _{x \rightarrow a^{+}}|f(x)-f(a)| w(x)=0, \\
\text { for each } a \in S^{-}, \quad \operatorname{ess}_{x \rightarrow a^{-}}|f(x)-f(a)| w(x)=0
\end{array}\right\} .
$$


Then

(a) The closure of $C(\mathbb{R}) \cap L^{\infty}(w)$ in $L^{\infty}(w)$ is $H_{0}$.

(b) If $w \in L_{\text {loc }}^{\infty}(\mathbb{R})$, then the closure of $C^{\infty}(\mathbb{R}) \cap L^{\infty}(w)$ in $L^{\infty}(w)$ is also $H_{0}$.

(c) If $\operatorname{supp}(w)$ is compact and $w \in L^{\infty}(\mathbb{R})$, then the closure of the space of polynomials is $H_{0}$ as well.

Theorem 2.1 is going to be an important tool which will allow us to obtain the key for the result about Hilbert extensions of Weierstrass' theorem with weights in the present paper.

\section{2. $\mathcal{G}$-valued functions.}

Definition 2.5. Let $\mathcal{G}$ be a real and separable Hilbert space and we consider any sequence $\left\{x_{n}\right\} \subset \mathcal{G}$. We say that the support of $\left\{x_{n}\right\}$ is the set of $n$ for which $x_{n} \neq 0$. We denote to support of $\left\{x_{n}\right\}$ by $\operatorname{supp}\left(x_{n}\right)$.

Let $\mathcal{G}$ be a real and separable Hilbert space. A $\mathcal{G}$-valued polynomial on $I$ is a function $\phi: I \rightarrow \mathcal{G}$, such that

$$
\phi(t)=\sum_{n \in \mathbb{N}} \xi_{n} t^{n},
$$

where $\left(\xi_{n}\right)_{n \in \mathbb{N}} \subset \mathcal{G}$ has finite support.

Let $\mathbb{P}(\mathcal{G})$ be the space of all $\mathcal{G}$-valued polynomials on $I$. It is well-known that $\mathbb{P}(\mathcal{G})$ is a subalgebra of the space $C(I ; \mathcal{G})$ of all continuous $\mathcal{G}$-valued functions on $I$.

For $1 \leq p \leq \infty, L_{\mathcal{G}}^{p}(I)$ denotes the set of all weakly measurable functions $f: I \rightarrow \mathcal{G}$ such that

$$
\int_{I}\|f(t)\|_{\mathcal{G}}^{p} d t<\infty, \text { if } 1 \leq p<\infty
$$

or

$$
\operatorname{ess} \sup _{t \in I}\|f(t)\|_{\mathcal{G}}<\infty, \text { if } p=\infty .
$$

Then $L_{\mathcal{G}}^{2}(I)$ is a Hilbert space with respect to the inner product

$$
\langle f, g\rangle_{L_{\mathcal{G}}^{2}(I)}=\int_{I}\langle f(t), g(t)\rangle_{\mathcal{G}} d t .
$$

$\mathbb{P}(\mathcal{G})$ is also dense in $L_{\mathcal{G}}^{p}(I)$, for $1 \leq p<\infty$. More details about these spaces may be found in [30].

Definition 2.6. Let $\mathcal{G}$ be a real and separable Hilbert space, a weight $w$ on $\mathcal{G}$ is a weakly measurable function $w: I \longrightarrow \mathcal{G}$. 
Definition 2.7. Let $w$ be a weight on $\mathcal{G}$, we define the space $L_{\mathcal{G}}^{\infty}(I, w)$ as the space of equivalence classes of all the $\mathcal{G}$-valued weakly measurable functions $f: I \longrightarrow \mathcal{G}$ with respect to the norm

$$
\|f\|_{L_{\mathcal{G}}^{\infty}(I, w)}:=\operatorname{ess}_{\sup _{t \in I}}\|(f w)(t)\|_{\mathcal{G}}
$$

where $f w: I \longrightarrow \mathcal{G}$ is defined as follows: If $\operatorname{dim} \mathcal{G}<\infty$; we have the functions $f$ and $w$ can be expressed by $f=\left(f_{1}, \ldots, f_{n_{0}}\right)$ and $w=$ $\left(w_{1}, \ldots, w_{n_{0}}\right)$, respectively, where $f_{j}, w_{j}: I \longrightarrow \mathbb{R}$, for $j=1, \ldots, n_{0}$, with $n_{0}=\operatorname{dim} \mathcal{G}$. Then

$$
(f w)(t):=\left(f_{1}(t) w_{1}(t), \ldots, f_{n_{0}}(t) w_{n_{0}}(t)\right), \text { for } t \in I .
$$

If $\operatorname{dim} \mathcal{G}=\infty$, let $\left\{\tau_{j}\right\}_{j \in \mathbb{Z}_{+}}$be a complete orthonormal system in $\mathcal{G}$, then for $t \in I$ the functions $f$ and $w$ can be expressed as $f(t)=$ $\sum_{j=0}^{\infty}\left\langle f(t), \tau_{j}\right\rangle_{\mathcal{G}} \tau_{j}$ and $w(t)=\sum_{j=0}^{\infty}\left\langle w(t), \tau_{j}\right\rangle_{\mathcal{G}} \tau_{j}$, respectively. So, we can define

$$
(f w)(t):=\sum_{j=0}^{\infty}\left\langle f(t), \tau_{j}\right\rangle_{\mathcal{G}}\left\langle w(t), \tau_{j}\right\rangle_{\mathcal{G}} \tau_{j}, \text { for } t \in I .
$$

In this way, we can study our approximation problem using the properties of commutative Banach algebra of $l^{2}(\mathbb{R})$.

The next proposition shows a result about algebraic properties and density of $\mathbb{P}(\mathcal{G})$ in $C(I ; \mathcal{G})$. The analogous result, when $\mathcal{G}$ is a complex separable Hilbert space, appeared in [30].

\section{Proposition 2.1.}

(i) $\mathbb{P}(\mathcal{G})$ is a subalgebra of the space of all $\mathcal{G}$-valued continuous functions on $I$.

(ii) The closure of $\mathbb{P}(\mathcal{G})$ in $L_{\mathcal{G}}^{\infty}(I)$ is $C(I ; \mathcal{G})$.

Proof. (i) It is straight forward.

(ii) It is enough to prove that $C(I ; \mathcal{G}) \subset \overline{\mathbb{P}(\mathcal{G})}$, since $\overline{\mathbb{P}(\mathcal{G})} \subset \overline{C(I ; \mathcal{G})}=$ $C(I ; \mathcal{G})$.

Case 1. $\operatorname{dim} \mathcal{G}<\infty$.

Let us assume that $\operatorname{dim} \mathcal{G}=n_{0}$. Given an orthonormal basis $\left\{\tau_{1}, \ldots, \tau_{n_{0}}\right\}$ of $\mathcal{G}, \epsilon>0$ and $f \in C(I ; \mathcal{G})=C\left(I, \mathbb{R}^{n_{0}}\right)$, then $f \sim\left(f_{1}, \ldots, f_{n_{0}}\right)$ with $f_{j} \in C(I), j=1, \ldots, n_{0}$. The Weierstrass' theorem guarantees that there exists $p_{k} \in \mathbb{P}$ such that

$$
\left\|f_{j}-p_{j}\right\|_{L^{\infty}(I)}<\frac{\epsilon}{\sqrt{n_{0}}}, \quad j=1, \ldots, n_{0} .
$$


If we consider the polynomial $p \in \mathbb{P}(\mathcal{G})$ such that $p \sim\left(p_{1}, \ldots, p_{n_{0}}\right)$, then we have that

$$
\begin{aligned}
& \|f-p\|_{L_{\mathcal{G}}^{\infty}(I)} \quad=\quad \operatorname{ess} \sup _{t \in I}\|(f-p)(t)\|_{\mathcal{G}} \\
& =\operatorname{ess}_{\sup }\left[\sum_{j=1}^{n_{0}}\left|\left\langle f(t)-p(t), \tau_{j}\right\rangle_{\mathcal{G}}\right|^{2}\right]^{1 / 2} \\
& \leq \operatorname{esssup}_{t \in I}\left\|\left(\left(f_{1}-p_{1}\right)(t), \ldots,\left(f_{n_{0}}-p_{n_{0}}\right)(t)\right)\right\|_{\mathbb{R}^{n_{0}}}<\epsilon .
\end{aligned}
$$

Case 2: $\mathcal{G}$ is infinite-dimensional.

Let $f \in C(I ; \mathcal{G})$ and $\left\{\tau_{j}\right\}_{j \in \mathbb{Z}_{+}}$a complete orthonormal system in $\mathcal{G}$, then for each $t \in I$

$$
f(t)=\sum_{j=0}^{\infty}\left\langle f(t), \tau_{j}\right\rangle_{\mathcal{G}} \tau_{j},
$$

consequently, given $\epsilon>0$ there exists $m_{0} \in \mathbb{Z}_{+}$such that

$$
\left\|f(t)-\sum_{j=0}^{n}\left\langle f(t), \tau_{j}\right\rangle \tau_{j}\right\|_{\mathcal{G}}<\epsilon, \text { whenever } n \geq m_{0} .
$$

Now, let us consider the functions $f_{j}: I \rightarrow \mathbb{R}$ given by $f_{j}(t)=\left\langle f(t), \tau_{j}\right\rangle_{\mathcal{G}}$. We have that $f \sim\left\{f_{j}\right\}$ with $\sum_{j \in \mathbb{Z}_{+}}\left|f_{j}(t)\right|^{2}<\infty$, for each $t \in I$ and $f_{j} \in C(I)$.

So, Weierstrass' approximation theorem guarantees that there exists a sequence $\left\{p_{j}\right\}_{j \in \mathbb{Z}_{+}} \subset \mathbb{P}$ such that

$$
\left\|f_{j}-p_{j}\right\|_{L^{\infty}(I)}<\frac{\epsilon}{j+1}, \quad j \in \mathbb{Z}_{+} .
$$

We define the $\mathcal{G}$-polynomials $\tilde{p}_{j} \in \mathbb{P}(\mathcal{G})$ by $\tilde{p}_{j}(t)=p_{j}(t) \tau_{j}$, for each $j \in \mathbb{Z}_{+}$. Then for $n \geq m_{0}$ we have

$$
\begin{aligned}
\left\|f(t)-\sum_{j=0}^{n} \tilde{p}_{j}(t)\right\|_{\mathcal{G}} & \leq\left\|f(t)-\sum_{j=0}^{n} f_{j}(t) \tau_{j}\right\|_{\mathcal{G}}+\left\|\sum_{j=0}^{n} f_{j}(t) \tau_{j}-\sum_{j=0}^{n} \tilde{p}_{j}(t)\right\|_{\mathcal{G}} \\
& \leq \epsilon+\left(\sum_{j=0}^{\infty}\left|f_{j}(t)-p_{j}(t)\right|^{2}\right)^{1 / 2} \\
& <\epsilon+\left(\sum_{j=0}^{\infty}\left(\frac{\epsilon}{j+1}\right)^{2}\right)^{1 / 2}=\epsilon\left(1+\left(\sum_{j=0}^{\infty} \frac{1}{(j+1)^{2}}\right)^{1 / 2}\right) .
\end{aligned}
$$


From these inequalities we can deduce that for a large enough $n$ there exists $q_{n}(t)=\sum_{j=0}^{n} \tilde{p}_{j}(t)$ such that

$$
\left\|f-q_{n}\right\|_{L_{\mathcal{G}}^{\infty}(I)}<C \epsilon .
$$

This completes the proof.

\section{Approximation in $L_{\mathcal{G}}^{\infty}(I, w)$}

In this section, we only deal with weights $w$ such that $\operatorname{supp}(w)=I$.

Definition 3.1. Let $\mathcal{G}$ be a real and separable Hilbert space and let $w$ be a weight on $\mathcal{G}$. We say that $w$ is admissible ${ }^{\star}$ if one of the following conditions is satisfied

i) If $\operatorname{dim} \mathcal{G}<\infty$ then each one of the components $w_{j}, 1 \leq j \leq \operatorname{dim} \mathcal{G}$, is a scalar weight.

ii) If $\operatorname{dim} \mathcal{G}=\infty$, let $\left\{\tau_{j}\right\}_{j \in \mathbb{Z}_{+}}$be a complete orthonormal system in $\mathcal{G}$, and $w(t)=\sum_{j=0}^{\infty}\left\langle w(t), \tau_{j}\right\rangle_{\mathcal{G}} \tau_{j}$, then each one of the functions $\left\langle w(t), \tau_{j}\right\rangle_{\mathcal{G}}$ is a scalar weight.

Let us observe that if $\operatorname{dim} \mathcal{G}=\infty$ and $w$ is admissible ${ }^{\star}$, then it induces a family of weighted $l^{2}(\mathbb{R})$ spaces, $\left\{l_{t}^{2}(\mathbb{R} ; w): t \in I\right\}$ given by

$$
l_{t}^{2}(\mathbb{R} ; w)=\left\{\left\{x_{j}\right\}_{j \in \mathbb{Z}_{+}}: \sum_{j=0}^{\infty}\left\langle w(t), \tau_{j}\right\rangle_{\mathcal{G}}\left|x_{j}\right|^{2}<\infty\right\} .
$$

For each $t \in I$, the function $w_{j}(t)=\left\langle w(t), \tau_{j}\right\rangle_{\mathcal{G}}$ also induces a linear isometry

$$
\begin{aligned}
& \Psi_{w_{j}}^{t}: l_{t}^{2}\left(\mathbb{R} ; w_{j}\right) \rightarrow l^{2}(\mathbb{R}) \text { given by } \\
& \qquad \Psi_{w_{j}}^{t}\left(\left\{x_{j}\right\}_{j \in \mathbb{Z}_{+}}\right)=\left\{w_{j}(t) x_{j}\right\}_{j \in \mathbb{Z}_{+}}=\left\{\left\langle w(t), \tau_{j}\right\rangle_{\mathcal{G}} x_{j}\right\}_{j \in \mathbb{Z}_{+}} .
\end{aligned}
$$

The reader is referred to [8] where weighted $l^{2}(\mathbb{R})$ spaces are studied. In order to characterize the $\mathcal{G}$-valued functions which can be approximated in $L_{\mathcal{G}}^{\infty}(I, w)$ by functions in $C(I ; \mathcal{G}) \cap L_{\mathcal{G}}^{\infty}(I, w)$, our argument requires an admissible* weight $w$. It is clear that in the one-dimensional case an admissible* weight is an arbitrary scalar weight on $I$, and therefore Theorem 2.1 in [26] holds in this case. 
Theorem 3.1. Let $\mathcal{G}$ be a real and separable Hilbert space and let $w$ be an admissible ${ }^{\star}$ weight on $\mathcal{G}$. Let us define

$H:=\left\{\begin{array}{c}f \in L_{\mathcal{G}}^{\infty}(I, w): \\ f \sim\left(f_{1}, \ldots, f_{n_{0}}\right) \text { and } f_{j} \in H_{j}, 1 \leq j \leq n_{0} \text { with } n_{0}=\operatorname{dim} \mathcal{G}, \\ \text { or } f \sim\left\{f_{j}\right\} \text { and } f_{j} \in H_{j}, j \in \mathbb{Z}_{+} \text {if } \operatorname{dim} \mathcal{G}=\infty .\end{array}\right\}$,

where

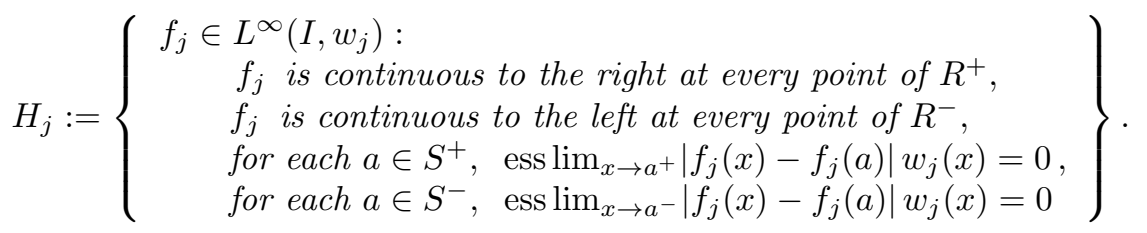

Then the closure of $C(I ; \mathcal{G}) \cap L_{\mathcal{G}}^{\infty}(I, w)$ in $L_{\mathcal{G}}^{\infty}(I, w)$ is $H$. Furthermore, if $w \in L_{\mathcal{G}}^{\infty}(I)$ then the closure of the space of $\mathcal{G}$-valued polynomials is $H$ as well.

Proof. Let us assume first that $\operatorname{dim} \mathcal{G}=n_{0}$. If $f \in$ ${\overline{C(I ; \mathcal{G}) \cap L_{\mathcal{G}}^{\infty}(I, w)}}^{L_{\mathcal{G}}^{\infty}(I, w)}$, then $f \sim\left(f_{1}, \ldots, f_{n_{0}}\right)$, with $f_{j}: I \longrightarrow \mathbb{R}$, $1 \leq j \leq n_{0}$. Given $\epsilon>0$, there exists $g \in C(I ; \mathcal{G}) \cap L_{\mathcal{G}}^{\infty}(I, w)$ such that $\|f-g\|_{L_{\mathcal{G}}^{\infty}(I, w)}<\epsilon$. Let us consider $\left(g_{1}, \ldots, g_{n_{0}}\right)$ such that $g_{j} \in C(I) \cap L^{\infty}\left(I, w_{j}\right)$ and $g \sim\left(g_{1}, \ldots, g_{n_{0}}\right)$, then

$$
\left|\left(f_{j}(t)-g_{j}(t)\right) w_{j}(t)\right| \leq \operatorname{ess}_{\sup }\left[\sum_{j=1}^{n_{0}}\left|\left(f_{j}(s)-g_{j}(s)\right) w_{j}(s)\right|^{2}\right]^{1 / 2} \text { a.e. }
$$

On other hand, $\left[\sum_{j=1}^{n_{0}}\left|\left(f_{j}(s)-g_{j}(s)\right) w_{j}(s)\right|^{2}\right]^{1 / 2}=\|f-g\|_{L_{\mathcal{G}}^{\infty}(I, w)}$, as consequence of $\mathcal{G}$ is isomorphic to $\mathbb{R}^{n_{0}}$ and the Parseval identity (see [8] or [32]). Then,

$$
\left\|f_{j}-g_{j}\right\|_{L^{\infty}\left(I, w_{j}\right)} \leq\|f-g\|_{L_{\mathcal{G}}^{\infty}(I, w)}<\epsilon .
$$

Hence, $f_{j} \in \overline{C(I) \cap L^{\infty}\left(I, w_{j}\right)} L^{L^{\infty}\left(I, w_{j}\right)}$ for $1 \leq j \leq n_{0}$, and the part (a) of Theorem 2.1 gives that $H$ contains $\overline{C(I ; \mathcal{G}) \cap L_{\mathcal{G}}^{\infty}(I, w)} L_{\mathcal{G}}^{\infty}(I, w)$.

In order to see that $H$ is contained in $\overline{C(I ; \mathcal{G}) \cap L_{\mathcal{G}}^{\infty}(I, w)}{ }^{L_{\mathcal{G}}^{\infty}(I, w)}$, let us fix $f \in H$ and $\epsilon>0$, and let us consider each one of its component functions $f_{j} \in H_{j}, j=1, \ldots, n_{0}$. By the part (a) of Theorem 2.1, there exists $g_{j} \in C(I) \cap L^{\infty}\left(I, w_{j}\right), j=1, \ldots, n_{0}$, such that

$$
\left\|f_{j}-g_{j}\right\|_{L^{\infty}\left(I, w_{j}\right)}<\frac{\epsilon}{\sqrt{n_{0}}}
$$


We consider the function $g \in C(I ; \mathcal{G})$ such that $g \sim\left(g_{1}, \ldots, g_{n_{0}}\right)$, then

$$
\begin{aligned}
& \|f-g\|_{L_{\mathcal{G}}^{\infty}(I, w)}=\operatorname{ess}_{\sup } \operatorname{sut}_{t \in I}\|((f-p) w)(t)\|_{\mathcal{G}} \\
& =\operatorname{ess}_{\sup }\left[\sum_{j=1}^{n_{0}}\left|\left(f_{j}(t)-g_{j}(t)\right) w_{j}(t)\right|^{2}\right]^{1 / 2}<\epsilon .
\end{aligned}
$$

If $w \in L_{\mathcal{G}}^{\infty}(I)$, the closure of the $\mathcal{G}$-valued polynomials is $H$ as well, as a consequence of Proposition 2.1.

In a similar way, if $\operatorname{dim} \mathcal{G}=\infty,\left\{\tau_{j}\right\}_{j \in \mathbb{Z}_{+}}$is a complete orthonormal system in $\mathcal{G}$ and $f \in \overline{C(I ; \mathcal{G}) \cap L_{\mathcal{G}}^{\infty}(I, w)}{ }^{L_{\mathcal{G}}^{\infty}(I, w)}$, then $f(t)=$ $\sum_{j=0}^{\infty}\left\langle f(t), \tau_{j}\right\rangle \tau_{j}$. Given $\epsilon>0$, there exists $g \in C(I ; \mathcal{G}) \cap L_{\mathcal{G}}^{\infty}(I, w)$ such that $\|f-g\|_{L_{\mathcal{G}}^{\infty}(I, w)}<\epsilon$. Let us consider $\left\{g_{j}\right\}_{j \in \mathbb{Z}_{+}}$such that $g_{j} \in$ $C(I) \cap L^{\infty}\left(I, w_{j}\right)$ and $g \sim\left\{g_{j}\right\}_{j \in \mathbb{Z}_{+}}$, then

$$
\left|\left(f_{j}(t)-g_{j}(t)\right) w_{j}(t)\right| \leq \operatorname{ess} s u p_{s \in I}\left[\sum_{j=0}^{\infty}\left|\left(f_{j}(s)-g_{j}(s)\right) w_{j}(s)\right|^{2}\right]^{1 / 2} \text { a.e. }
$$

On other hand, $\left[\sum_{j=1}^{\infty}\left|\left(f_{j}(s)-g_{j}(s)\right) w_{j}(s)\right|^{2}\right]^{1 / 2}=\|f-g\|_{L_{\mathcal{G}}^{\infty}(I, w)}$, as consequence of $\mathcal{G}$ is isomorphic to $l^{2}(\mathbb{R})$ and the Parseval identity (see [8] or [32]). Then,

$$
\|\left(f_{j}-g_{j}\left\|_{L^{\infty}\left(I, w_{j}\right)} \leq\right\| f-g \|_{L_{\mathcal{G}}^{\infty}(I, w)}<\epsilon .\right.
$$

Hence, $f_{j} \in{\overline{C(I) \cap L^{\infty}\left(I, w_{j}\right)}}^{L^{\infty}\left(I, w_{j}\right)}$ for $j \in \mathbb{Z}_{+}$, and the part (a) of Theorem 2.1 gives that $H$ contains $\overline{C(I ; \mathcal{G}) \cap L_{\mathcal{G}}^{\infty}(I, w)} L_{\mathcal{G}}^{\infty}(I, w)$.

In order to see that $H$ is contained in $\frac{G(\mathcal{G}) \cap L_{\mathcal{G}}^{\infty}(I, w)}{C\left(L_{\mathcal{G}}^{\infty}(I, w)\right.}$, let $f \in H$ and $\epsilon>0$, and let us consider the component functions $f_{j} \in H_{j}$ of $f, 0 \leq j<\infty$. Since $w_{j}(t)=\left\langle w(t), \tau_{j}\right\rangle_{\mathcal{G}}$ is a weight, by the part (a) of Theorem 2.1, there exists $g_{j} \in C(I) \cap L^{\infty}\left(I, w_{j}\right), 0 \leq j<\infty$, such that

$$
\left\|f_{j}-g_{j}\right\|_{L^{\infty}\left(I, w_{j}\right)}<\frac{\epsilon}{j+1}, \quad j \in \mathbb{Z}_{+} .
$$


We define the function $g: I \longrightarrow \mathcal{G}$ by $g(t)=\sum_{j=0}^{\infty} g_{j}(t) \tau_{j}$, then

$$
\begin{aligned}
& \|f-g\|_{L_{\mathcal{G}}^{\infty}(I, w)}=\operatorname{ess}_{\sup _{t \in I}}\|((f-g) w)(t)\|_{\mathcal{G}} \\
& =\operatorname{ess}_{\sup } \operatorname{sit}_{t \in I}\left\|\left\{\left(f_{j}(t)-g_{j}(t)\right) w_{j}(t)\right\}\right\|_{l^{2}(\mathbb{R})} \\
& =\operatorname{ess}_{\sup }\left[\sum_{j=0}^{\infty}\left|f_{j}(t)-g_{j}(t)\right|^{2} w_{j}^{2}(t)\right]^{1 / 2} \\
& \leq\left[\sum_{j=0}^{\infty}\left(\frac{\epsilon}{j+1}\right)^{2}\right]^{1 / 2}=\epsilon\left[\sum_{j=0}^{\infty} \frac{1}{(j+1)^{2}}\right]^{1 / 2}
\end{aligned}
$$

This result is similar when $\mathcal{G}$ is a complex separable Hilbert space and it can also be extended to $L_{L(\mathcal{G})}^{\infty}(I, w)$, where $L(\mathcal{G})$ is the space of operators on $\mathcal{G}$.

Acknowledgment. The author wishes to thank the Professors José Manuel

Rodríguez and Stephen Andrea for their suggestions and comments which have improved the presentation of the paper.

\section{References}

[1] B. Baillaud and H. Bourget, Correspondence d'Hermite et de Stieltjes, Gauthier-Villars, Paris, Tome II, 1905.

[2] R. G. Bartle, The elements of Real Analysis, John Wiley \& Sons, Inc. (2nd ed.), New York, 1976.

[3] E. Bishop, A generalization of the Stone-Weierstrass theorem, Pacific J. Math., 11 (1961), 777-783.

[4] R. C. Brown and B. Opic, Embeddings of weighted Sobolev spaces into spaces of continuous functions, Proc. R. Soc. Lond. A, 439 (1992), 279296.

[5] E. W. Cheney, Introduction to Approximation Theory, McGraw-Hill Book Company, New York, 1966.

[6] W. W. Comfort, Edwin Hewitt as Topologist: An Appreciation, Topology Atlas, http://at.yorku.ca/topology, (2001).

[7] B. Della Vecchia, G. Mastroianni and J. Szabados, Approximation with exponential weights in $[-1,1]$, J. Math. Anal. Appl., 272 (2002), 1-18. 
212 On Hilbert extensions of Weierstrass' theorem with weights

[8] F. Deutsch, Best Approximation in Inner Product Spaces, SpringerVerlag, New York, 2001.

[9] N. Dinculeanu, Vector Measures, Pergamon Press Ltd., Headington Hill Hall, Oxford, 1967.

[10] H. Gingold, Approximation of unbounded functions via compactification, J. Approx. Theory, 131 (2004), 284-305.

[11] E. Hewitt, Certain generalizations of the Weierstrass approximation theorem, Duke Math. J., 14 (1947), 419-427.

[12] E. Hewitt and H. S. Zuckerman, Approximation by polynomials with integral coefficients, a reformulation of the Stone-Weierstrass theorem, Duke Math. J., 26 (1959), 305-324.

[13] T. Husain, Orthogonal Schauder Bases. Marcel Dekker, Inc. (1st ed.), New York, 1990.

[14] A. Kufner and B. Opic, How to define reasonably Weighted Sobolev Spaces, Commentationes Mathematicae Universitatis Caroline 25 (1984), 537-554.

[15] D. S. Lubinsky Weierstrass' Theorem in the twentieth century: a selection, Quaestiones Mathematicae, 18 (1995), 91-130.

[16] D. S. Lubinsky, Bernstein's weighted approximation on $\mathbb{R}$ still has problems, Electronic Transactions on Numerical Analysis, 25 (2006), $166-177$.

[17] D. S. Lubinsky, A survey of weighted polynomial approximation with exponential weights, Surveys in Approximation Theory, 3 (2007), 1105.

[18] L. Nachbin, Elements of Approximation Theory, D. Van Nostrand Company Inc., New York, 1967.

[19] D. Pérez and Y. Quintana, A survey on the Weierstrass approximation theorem, Divulgaciones Matemáticas, 16 (2008), 231-247.

[20] H. Pijeira, Y. Quintana and W. Urbina, Zero localization and asymptotic behavior of orthogonal polynomials of Jabobi-Sobolev, Rev. Col. Mat., 35 (2001), 77-97.

[21] A. Pinkus, Weierstrass and Approximation Theory, J. Approx. Theory, 107 (2000), 1-66.

[22] A. Pinkus, Density Methods and results in Approximation Theory, Banach Center Publications, 64 (2004).

[23] A. Portilla, Y. Quintana, J. M. Rodríguez and E. Tourís, Weierstrass' Theorem with weights, J. Approx. Theory, 127 (2004), 83-107. 
[24] A. Portilla, Y. Quintana, J. M. Rodríguez and E. Tourís, Weierstrass' theorem in weighted Sobolev spaces with $k$ derivatives: announcement of results, Electronic Transactions on Numerical Analysis 24 (2006), 103-107.

[25] A. Portilla, Y. Quintana, J. M. Rodríguez and E. Tourís, Weierstrass' theorem in weighted Sobolev spaces with $k$ derivatives, Rocky Mountain J. Math., 37 (2007), 1989-2024.

[26] A. Portilla, Y. Quintana, J. M. Rodríguez and E. Tourís, Weighted Weierstrass' Theorem with first derivatives, J. Math. Anal. Appl., 334 (2007), 1667-1698.

[27] J. M. Rodríguez, Weierstrass' Theorem in weighted Sobolev spaces, J. Approx. Theory, 108 (2001), 119-160.

[28] W. Rudin, Real and Complex Analysis (Second Edition), McGraw-Hill, 1974.

[29] M. H. Stone, The Generalized Weierstrass Approximation Theorem, Mathematics Magazine 21 (1947 /1948), 167-184/237-254. Reprinted in : MAA Studies in Mathematics, 1, R. C. Buck, editor, Math. Assn. America, (1962).

[30] B. Sz.-Nagy, C. Foias, Analyse Harmonique des Opérateurs de L'espace de Hilbert, Masson et Cie. Akadémiai Kiadó. Hongrie, 1966.

[31] G. Szegö, Orthogonal Polynomials, Coll. Publ. Amer. Math. Soc., Vol. 23, (4th ed.), Providence, R.I., 1975.

[32] N. Young, An introduction to Hilbert spaces, Cambridge University Press, hardback edition. Reprinted (1995).

Departamento de Matemáticas

Puras y Aplicadas

Edificio Matemáticas y Sistemas (MYS)

Apartado Postal: 89000, Caracas 1080 A

Universidad Simón Bolívar

Caracas

Venezuela

(E-mail : yquintana@usb.ve) 


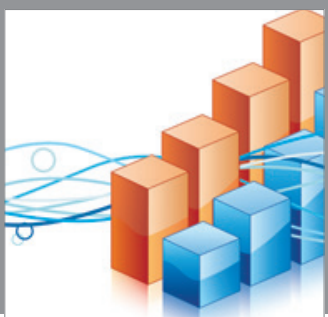

Advances in

Operations Research

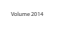

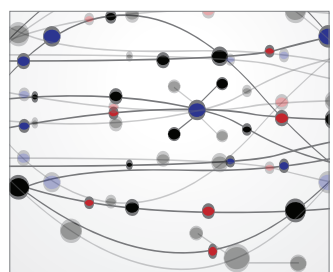

\section{The Scientific} World Journal
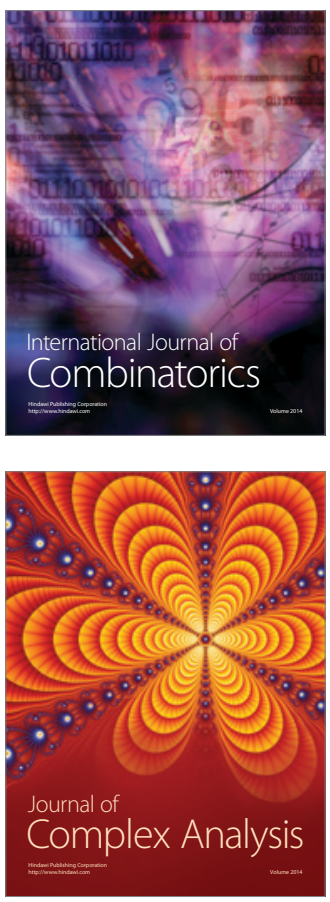

International Journal of

Mathematics and

Mathematical

Sciences
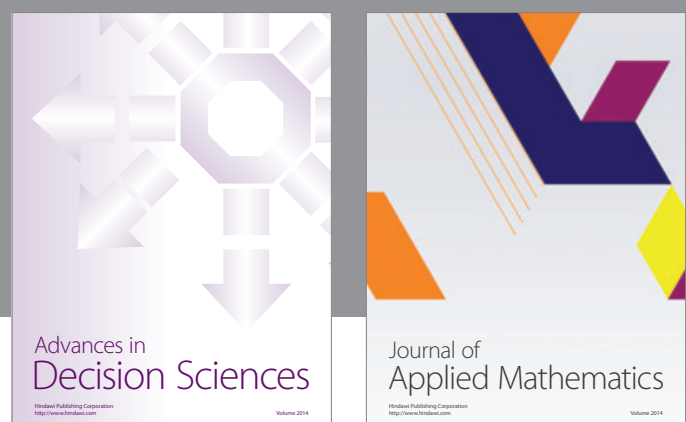

Journal of

Applied Mathematics
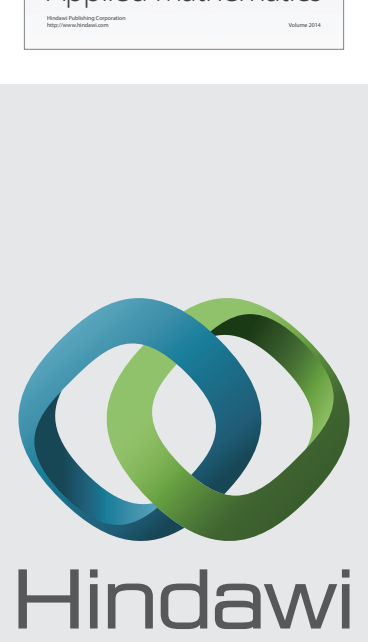

Submit your manuscripts at http://www.hindawi.com
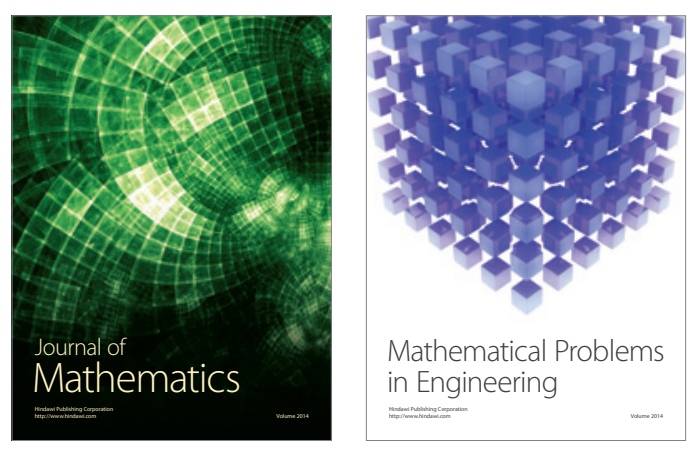

Mathematical Problems in Engineering
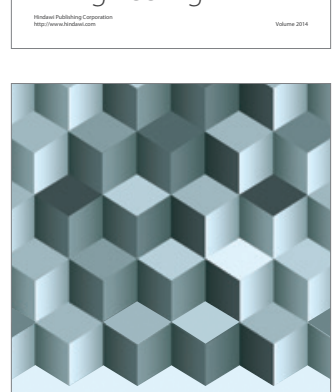

Journal of

Function Spaces
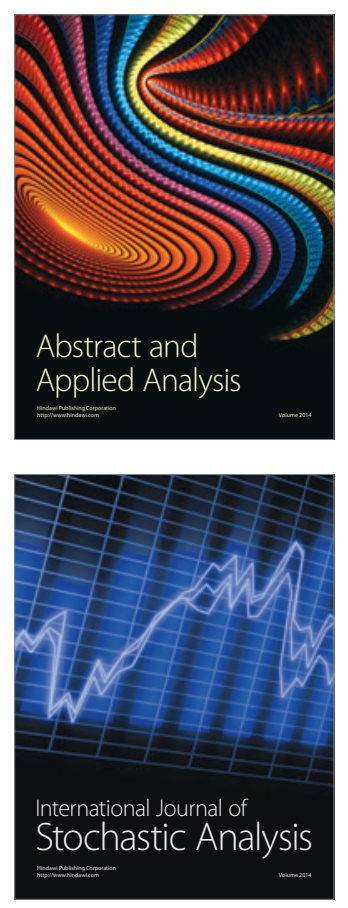

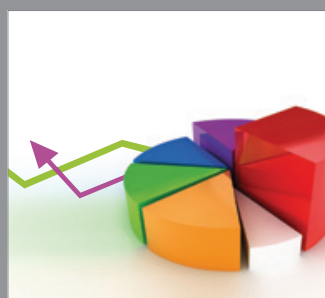

ournal of

Probability and Statistics

Promensencen
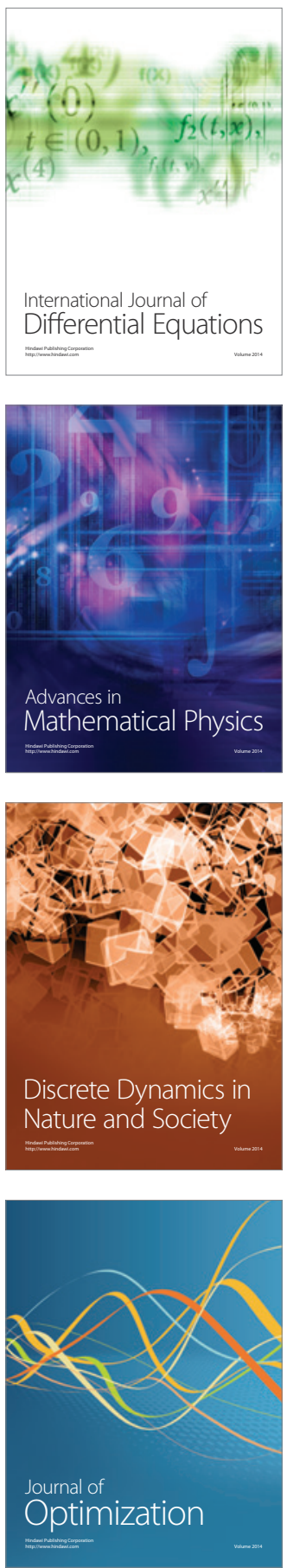